\title{
Dependence of gas-phase crotonaldehyde hydrogenation selectivity and activity on the size of Pt nanoparticles (1.7 - 7.1 nm) supported on SBA-15
}

\author{
Michael E. Grass ${ }^{\dagger}$, Robert M. Rioux ${ }^{\ddagger}$, Gabor A. Somorjai* \\ Lawrence Berkeley National Laboratory and Department of Chemistry, University of \\ California, Berkeley, California 94720 \\ ${ }^{\dagger}$ Current Address: Advanced Light Source, Lawrence Berkeley National Laboratory, \\ Berkeley, CA 94720 \\ Current address: Department of Chemical Engineering, Pennsylvania State University, \\ University Park, PA 16802-4400
}

*To whom correspondence should be addressed: somorjai@berkeley.edu

Keywords: crotonaldehyde, hydrogenation, platinum, nanoparticles, selectivity, decarbonylation

Running title: Influence of particle size on selectivity during crotonaldehyde hydrogenation 


\begin{abstract}
The selectivity and activity for the hydrogenation of crotonaldehyde to crotyl alcohol and butyraldehyde was studied over a series of Pt nanoparticles (diameter of 1.7, 2.9, 3.6, and $7.1 \mathrm{~nm}$ ). The nanoparticles were synthesized by the reduction of chloroplatinic acid by alcohol in the presence of poly(vinylpyrrolidone) (PVP), followed by encapsulation into mesoporous SBA-15 silica. The rate of crotonaldehyde hydrogenation and selectivity towards crotyl alcohol both increase with increasing particle size. , The selectivity towards crotyl alcohol increased from $13.7 \%$ to $33.9 \%$ (8 Torr crotonaldehyde, 160 Torr $\mathrm{H}_{2}$ and $353 \mathrm{~K}$ ), while the turnover frequency increases from 2.1 $\times 10^{-2} \mathrm{~s}^{-1}$ to $4.8 \times 10^{-2} \mathrm{~s}^{-1}$ with an increase in the particle size from $1.7 \mathrm{~nm}$ to $7.1 \mathrm{~nm}$. The decarbonylation pathway to form propene and $\mathrm{CO}$ is enhanced over the higher proportion of coordinatively unsaturated sites on the smaller nanoparticles. The apparent activation energy remains constant $\left(\sim 16 \mathrm{kcal} \mathrm{mol}^{-1}\right.$ for the formation of butyraldehyde and $\sim 8 \mathrm{kcal}$ $\mathrm{mol}^{-1}$ for the formation of crotyl alcohol) as a function of particle size. In the presence of 130 - 260 mTorr $\mathrm{CO}$, the reaction rate decreases for all products with a $\mathrm{CO}$ reaction order of -0.9 for crotyl alcohol and butyraldehyde over $7.1 \mathrm{~nm}$ Pt particles; over $1.7 \mathrm{~nm} \mathrm{Pt}$ particles, the order in $\mathrm{CO}$ is -1.4 and -0.9 , respectively. Hydrogen reduction at $673 \mathrm{~K}$ after calcination in oxygen results in increased activity and selectivity relative to reduction at either higher or lower temperature; this is discussed with regards to the incomplete removal and/or change in morphology of the polymeric surface stabilizing agent, poly(vinylpyrrolidone) used for the synthesis of the Pt nanoparticles..
\end{abstract}




\section{Introduction}

Polymer and surfactant stabilized transition metal nanoparticles (NPs) have been utilized as both homogeneous and heterogeneous catalysts over the past decade [1]. Their catalytic ability has been studied extensively both as colloidal suspensions [2] and as oxide- or carbon-supported NPs $[3,4]$. The polymer mediated method of metal colloid synthesis can yield transition metal NPs with narrow particle size distribution and has the potential to serve as model systems for the elucidation of particle size effects in heterogeneous catalysis $[5,6]$.

In our previous work, we developed two strategies for supporting poly(vinylpyrrolidone) (PVP)-protected Pt nanoparticles on mesoporous SBA-15 silica and studied the particle size dependence of the single product reactions (ethylene hydrogenation and ethane hydrogenolysis) and the influence of particle size on the poisoning of ethylene hydrogenation by carbon monoxide [5-9]. In this paper, we report on the selective hydrogenation of crotonaldehyde to crotyl alcohol and butyraldehyde over a series of $\operatorname{Pt}(X) / \mathrm{SBA}-15(X=1.7,2.9,3.6$, and $7.1 \mathrm{~nm})$ catalysts prepared by nanoparticle encapsulation (NE) [5]. We examined the effect of particle size on both the overall conversion of crotonaldehyde and the selectivity towards the industrially desired unsaturated alcohol, crotyl alcohol. We demonstrate NE-synthesized catalysts have similar catalytic activity and selectivity for crotonaldehyde hydrogenation as conventionally-prepared (incipient wetness, ion-exchange, etc.) catalysts [10].

The hydrogenation of crotonaldehyde and other $\alpha, \beta$-unsaturated aldehydes is of fundamental scientific interest because of the two distinct unsaturated chemical bonds $(\mathrm{C}=\mathrm{C}$ and $\mathrm{C}=\mathrm{O})$ are present in the same molecule, serving as a useful probe molecule for 
studies of heterogeneous catalytic chemoselectivity (Scheme 1). Gallezot and Richard summarized many of the properties that determine catalytic activity and selectivity for the conversion of $\alpha, \beta$-unsaturated aldehydes by heterogeneous catalysts [10]. Properties of the catalyst that influence both activity and selectivity include the type of metal, the exposed crystal faces of the extended single-crystal or nanoparticle surface, adsorbed ligands (e.g. organometallic tin) and promoters (e.g. K, Fe, Sn) which modify (electronically or geometrically) the surface of the metallic nanoparticle. The structure of the $\alpha, \beta$-unsaturated aldehyde influences reaction selectivity. Bulky side groups such as the phenyl group adjacent to the aliphatic double bond in cinnamaldehyde or the pair of methyl groups adjacent to the aliphatic double bond in 3-methyl-crotonaldehyde lead to higher selectivity to the unsaturated alcohol because of steric hindrance of the $\mathrm{C}=\mathrm{C}$ double bond. In the case of crotonaldehyde, the $\mathrm{C}=\mathrm{C}$ double bond is flanked by a methyl group only, and the selective hydrogenation of the carbonyl group over the $\mathrm{C}=\mathrm{C}$ double bond becomes significantly more challenging [10].

In previous investigations of the influence of particle size on the overall turnover frequency (TOF) and selectivity for crotyl alcohol from crotonaldehyde, it was observed that both generally increase with increasing particle size [11-13]. The role of particle size appears to be three-fold. The first is related to the fraction of surface atoms in close packed (111) planes versus atoms at lower coordination sites. Crotonaldehyde adsorbs preferentially via a di- $\sigma$ bond between the $\mathrm{C}=\mathrm{O}$ and the $\mathrm{Pt}(111)$ surface, while on the (100) surface, it bonds to the surface in a flat $\eta^{4}$ configuration through both of its $\pi$-bonds [14]. On the (110) surface, the most corrugated of the three lowest energy fcc surfaces, crotonaldehyde bonds via the $\mathrm{C}=\mathrm{C} \pi$-bond [14]. The increase in selective $\mathrm{C}=\mathrm{O}$ 
interaction on (111) facets is responsible for the increased selectivity towards the unsaturated alcohol. Larger nanoparticles have a larger fraction of surface atoms in terraces with a close-packed structure than do smaller particles and therefore tend to be more selective for the formation of crotyl alcohol [13]. The second particle size effect is the steric hindrance of the $\mathrm{C}=\mathrm{C}$ bond, which is more easily accommodated on smaller NPs, thus decreasing selectivity towards the unsaturated alcohol over such catalysts. The third effect is decreased catalytic activity over smaller NPs due to decarbonylation of crotonaldehyde to $\mathrm{CO}$ and $\mathrm{C}_{3}$ hydrocarbons as well as other $\mathrm{C}-\mathrm{C}$ bond breaking reactions occur preferentially on the corner and edge sites that are more prevalent on smaller particles. Adsorbed CO (from decarbonylation) and the carbon fragments deposited due to $\mathrm{C}-\mathrm{C}$ bond breaking poison the catalyst surface [15].

For the investigation of gas-phase heterogeneous catalysis using polymer encapsulated NPs on oxide supports, the organic capping molecules are typically removed by thermal treatment (pyrolysis, combined calcination and reduction) [6, 16, 17]. In this investigation, we consider the effects of such thermal treatments on the final state of the surface protecting polymer (PVP) and its implication on catalysis.

\section{Experimental}

\subsection{Catalyst Synthesis}

The preparation of the Pt nanoparticles (NPs) used in this study and their anchoring in mesoporous SBA-15 silica by nanoparticle encapsulation (NE) has been reported previously [5, 6]. In this study, a series of $\operatorname{Pt}(X) / \mathrm{SBA}-15(X=1.7-7.1 \mathrm{~nm})$ were synthesized with nominal loadings of $1-3 \%$. Higher weight loading of Pt was used for the catalysts containing larger NPs to account for the decrease in the total surface area of 
larger NPs compared to catalysts containing smaller nanoparticles at comparable weight loading. The 1.7 and $7.1 \mathrm{~nm}$ NPs were synthesized by the polyol reduction process in ethylene glycol (EG) $[6,18,19]$, while 2.9 and $3.6 \mathrm{~nm}$ particles were synthesized in an $80 / 20 \mathrm{v} / \mathrm{v}$ mixture of methanol and water by alcohol reduction $[6,20]$. The $3.6 \mathrm{~nm}$ particles were synthesized by a seeded growth method using the as-synthesized PVPprotected $2.9 \mathrm{~nm}$ particles as the seeds [20]. All of the NPs except for the $1.7 \mathrm{~nm}$ particles were synthesized in the presence of PVP, which sterically protects the particles from aggregation [21]. The $1.7 \mathrm{~nm}$ particles are stabilized electrostatically in a $\mathrm{NaOH}$ solution in EG during synthesis, then precipitated from the EG solution and transferred to a volume of ethanol containing PVP.

The nanoparticles were incorporated into silica by synthesizing the mesoporous SBA15-like silica matrix in neutral $\mathrm{pH}$ under hydrothermal conditions in the presence of the PVP-protected NPs [5]. The catalysts were washed in ethanol, dried in air at $373 \mathrm{~K}$ and calcined ex-situ in $\mathrm{O} 2$ at $723 \mathrm{~K}$ for $12 \mathrm{~h}(\mathrm{Pt}(2.9 \mathrm{~nm}) / \mathrm{SBA}-15$ and $\mathrm{Pt}(3.6 \mathrm{~nm}) / \mathrm{SBA}-15)$, at $623 \mathrm{~K}$ for $12 \mathrm{~h}(\mathrm{Pt}(1.7 \mathrm{~nm}) / \mathrm{SBA}-15)$, or at $723 \mathrm{~K}$ for $24 \mathrm{~h}(\mathrm{Pt}(7.1 \mathrm{~nm}) / \mathrm{SBA}-15)$ [6]. After calcination, the catalysts were characterized by X-ray diffraction (XRD) with a Bruker D8 GADDS diffractometer with Co $\mathrm{K}_{\alpha}$ radiation $(\lambda=1.79 \AA)$ and transmission electron microscopy (TEM, Philips/FEI Tecnai 12 microscope, $100 \mathrm{kV}$ operating voltage) for confirmation of the bulk particle size. The total surface area and metallic surface area were measured by $\mathrm{N}_{2}$ physisorption (BET method) and total $\mathrm{H}_{2}$ chemisorption with a Quantachrome Autosorb 1 automated sorption analyzer. The catalysts were reduced insitu prior to chemisorption by the same protocol used for the reduction of catalysts in reaction studies described below ( $673 \mathrm{~K}$ in $\mathrm{H}_{2}$ for $2 \mathrm{~h}$ ) followed by evacuation at $623 \mathrm{~K}$ 
for $1 \mathrm{~h}$. The catalyst was then cooled to $303 \mathrm{~K}$ under vacuum followed by volumetric $\mathrm{H}_{2}$ chemisorption. The total metal content was determined by inductively coupled plasma optical emission spectroscopy (ICP-OES) at Galbraith Laboratories (Knoxville, TN USA).

\subsection{Catalytic Studies}

Catalytic reactions were carried out in a stainless steel (1/8") flow reactor system equipped with mass flow controllers (MKS Instruments) for $\mathrm{He}, \mathrm{H}_{2}$, and $\mathrm{O}_{2}$ (UHP, Praxair, used as-received) and an inline Pyrex saturator for the introduction of crotonaldehyde (Sigma-Aldrich, 99.9\%, predominately trans) into the feed. The catalyst was reduced in situ at the desired temperature (typically $673 \mathrm{~K}$ ) for $2 \mathrm{~h}$ in $\mathrm{H}_{2}(160$ Torr)/He. After cooling to the reaction temperature, the flow of 160 Torr $\mathrm{H}_{2} / \mathrm{He}$ was passed through a saturator containing crotonaldehyde held at $273 \mathrm{~K}$ using an ice bath. The inlet conditions prior to contact with the catalyst bed was 8 Torr crotonaldehyde, 160 Torr $\mathrm{H}_{2}$ and balance He.

The catalyst was placed on top of a quartz frit in a 1/4” O. D. Pyrex U-tube reactor. For a typical experiment, $\sim 15 \mathrm{mg}$ catalyst was diluted with $\sim 50 \mathrm{mg}$ acid-washed and calcined low surface area quartz (Sigma Aldrich) and loaded into the reactor between beds of the same acid-washed and calcined quartz. The effluent stream was analyzed by gas chromatography (HP 5890 Series II) using a flame ionization detector (FID) for analysis of organics and a thermal conductivity detector (TCD) for $\mathrm{H}_{2}$ detection. The reaction was started at $353 \mathrm{~K}$ until a stable activity was measured (i.e. until initial deactivation was complete and a steady-state activity was reached); most catalysts required $\sim 2.5 \mathrm{~h}$ to reach steady state. The reaction temperature was then changed (and 
left for $1.5 \mathrm{~h}$ at each temperature) to $373,393,383,373,363$, and finally back to $353 \mathrm{~K}$ for a check on the extent of catalyst deactivation. Unless stated otherwise, data reported in this paper is the steady-state rate at $353 \mathrm{~K}$ (the deactivation check measurement). The activation energy for all samples was determined using the rates obtained while cooling from 393 to $353 \mathrm{~K}$. The conversion at each temperature was averaged from ten GC injections. The TOF was calculated by normalizing the reaction rate to the number surface Pt atoms based on TEM measurements and the Pt loading determined by ICPOES. Selectivity values were calculated by dividing the TOF a particular product by the overall TOF (the rate of disappearance of crotonaldehyde).

$\mathrm{CO}$ poisoning experiments were conducted at $353 \mathrm{~K}$ in the same manner described above, but with the addition of $1 \% \mathrm{CO}$ in $\mathrm{He}$ (Praxair, UHP) corresponding to $\mathrm{CO}$ partial pressures of $134-262$ mTorr in the feed stream.

\section{Results and Discussion}

\subsection{Catalyst Properties}

The as-synthesized NPs were analyzed by TEM and XRD before encapsulation and the SBA-15-supported Pt catalysts were characterized by ICP-OES, TEM, XRD, and chemisorption after calcination. An exhaustive analysis of the characteristics of these catalysts has been given previously and no notable deviations from the previous report were observed [5]. The metal loading determined by ICP-OES analysis, the metal dispersion determined from $\mathrm{H}_{2}$ chemisorption measurements, and a summary of the catalytic results are listed in Table 1 . The particle size determined from TEM and XRD line-broadening (determined using the Scherrer equation) are in agreement and analysis 
of the TEM images indicates a standard deviation in particle size of $\sim 10 \%$ for all catalysts.

The dispersion measured by total hydrogen chemisorption are $\sim 50 \%$ lower than the measured TEM results. The theoretical $\mathrm{H} / \mathrm{Pt}_{\mathrm{T}}$ (total $\mathrm{Pt}$ ) ratio for 1.7, 2.9, 3.6, and $7.1 \mathrm{~nm}$ Pt NPs are 0.66, 0.39, 0.31, and 0.16, respectively, assuming hemispherical particles and 1:1 stoichiometry between $\mathrm{H}$ and $\mathrm{Pt}_{\mathrm{s}}$ (surface $\mathrm{Pt}$ ). This discrepancy suggests that the ex situ calcination - in situ reduction procedure described in the experimental section produce catalysts capable of adsorbing hydrogen up to only $50 \%$ coverage relative to the total number of surface Pt atoms, regardless of particle size. The suppressed adsorption of hydrogen is likely due to blockage of surface sites by residual carbonaceous material from the decomposition of PVP during the oxygen and hydrogen pretreatment steps. The fractional coverage of this layer as measured by $\mathrm{H}_{2}$ chemisorption is independent of particle size. In a study by Du et al., PVP-protected Pt (2.5 nm) nanoparticles were heated in $\mathrm{N}_{2}$ for $0.5 \mathrm{~h}$ at $773 \mathrm{~K}$ : after the heat treatment, the molar $\mathrm{C}: \mathrm{Pt}_{\mathrm{s}}$ ratio was 35 (before thermal treatment, the ratio was 85) [22]. In this work, we have used oxygen pretreatment for a much longer time $(12-24 \mathrm{~h})$, but it appears that residual carbon from either PVP or P123 remains on the Pt surface. The surface coverage and chemical composition of this layer may play a significant role in the catalytic properties of the calcined/reduced Pt nanoparticles.

\subsection{Crotonaldehyde Hydrogenation}

Butyraldehyde formed by the selective hydrogenation of the $\mathrm{C}=\mathrm{C}$ bond in crotonaldehyde, is the major product of crotonaldehyde hydrogenation over all catalysts and the entire temperature range $(353-393 \mathrm{~K})$ studied. The minor products are crotyl 
alcohol (the second most abundant product) formed by selective hydrogenation of the $\mathrm{C}=\mathrm{O}$ bond, butanol (hydrogenation of both the $\mathrm{C}=\mathrm{C}$ and $\mathrm{C}=\mathrm{O}$ bonds), and propylene and propane (decarbonylation) (Scheme 1).

The initial time-on-stream data for $\operatorname{Pt}(1.7 \mathrm{~nm}) / \mathrm{SBA}-15$ and $\operatorname{Pt}(7.1 \mathrm{~nm}) / \mathrm{SBA}-15$ shows an initial deactivation period that lasts $\sim 2.5 \mathrm{~h}$. It is well-documented in the literature that this deactivation is a result of decarbonylation and the subsequent adsorption of $\mathrm{CO}$ on the Pt surface, as well as the deposition of carbon resulting from $\mathrm{C}-\mathrm{C}$ and $\mathrm{C}-\mathrm{H}$ bond scission [23-26]. $\mathrm{C}_{3}$ hydrocarbons produced from the decarbonylation pathway were detected at all reaction temperatures and on all catalysts, indicating the formation of $\mathrm{CO}$ (although $\mathrm{CO}$ was not detected in the gas-phase by gas chromatography). The initial TOF for decarbonylation is at least $10^{-3} \mathrm{~s}^{-1}$ for all four catalysts. If we assume that the $\mathrm{CO}$ formed by decarbonylation remains on the surface, enough $\mathrm{CO}$ forms to saturate the Pt surface sites (based on the number of surface atoms determined by TEM measurements) within 15-20 min after the start of the reaction. The deactivation of heterogeneous catalysts for the hydrogenation of $\alpha, \beta$-unsaturated aldehydes by $\mathrm{CO}$ as a result of decarbonylation has been revealed in surface science studies [26], infrared spectroscopy studies on supported catalysts [24], and kinetic studies [25] over a number of different transition metals. A second pathway for deactivation is coke formation from $\mathrm{C}-\mathrm{C}$ bond rupture. This process may be slower than the formation of $\mathrm{CO}$ by decarbonylation, but the products of this process will not desorb during reaction.

The temperature dependence for hydrogenation is similar for all catalysts studied. The overall apparent activation energy for the disappearance of crotonaldehyde is independent of the particle size (Table 1). The apparent activation energy for the 
individual products is also not dependent on particle size: $16-17 \mathrm{kcal} \mathrm{mol}^{-1}$ for $\mathrm{C}=\mathrm{C}$ hydrogenation to butyraldehyde, $7-10 \mathrm{kcal} \mathrm{mol}^{-1}$ for $\mathrm{C}=\mathrm{O}$ hydrogenation to crotyl alcohol, $10-15 \mathrm{kcal} \mathrm{mol}^{-1}$ for the complete hydrogenation to butanol, and $11-12 \mathrm{kcal}$ $\mathrm{mol}^{-1}$ for decarbonylation. The temperature dependence for all products catalyzed by $\operatorname{Pt}(7.1 \mathrm{~nm}) / \mathrm{SBA}-15$ is shown in Figure 1. Lower temperatures favor the formation of minor products (particularly crotyl alcohol) relative to butyraldehyde (the apparent activation energy for the formation of butyraldehyde is higher than for the formation of crotyl alcohol over all catalysts studied). Butyraldehyde is the more thermodynamicallyfavored product, but it is also the more kinetically-hindered (higher apparent activation energy). These observations are consistent with general trends of crotonaldehyde hydrogenation over Pt catalysts that have been reported previously [10].

\subsection{Particle Size Effects on Selectivity and Activity for Crotonaldehyde Hydrogenation}

The reaction selectivity to crotyl alcohol and steady-state TOF at $353 \mathrm{~K}$ for all catalysts is listed in Table 1 and shown in Figures 2 and 3. The overall rate and selectivity to crotyl alcohol increased linearly with increasing particle size. The steadystate rate increased by a factor of two (from $2.4 \times 10^{-2} \mathrm{~s}^{-1}$ to $4.8 \times 10^{-2} \mathrm{~s}^{-1}$ ) and the selectivity towards crotyl alcohol varied by a factor of 3 (from $13.7 \%$ to $33.9 \%$ ) with an increase in the particle size from 1.7 to $7.1 \mathrm{~nm}$. The increase in selectivity toward crotyl alcohol with particle size results mainly from the increased interaction of $\mathrm{C}=\mathrm{O}$ with terrace sites on Pt [10, 14, 27].

The initial deactivation is also dependent on particle size. During the first 10 minutes on stream, $\operatorname{Pt}(1.7 \mathrm{~nm}) / \mathrm{SBA}-15$ is 1.5 times more active than $\operatorname{Pt}(7.1 \mathrm{~nm}) / \mathrm{SBA}-15(6.9 \times$ $10^{-2} \mathrm{~s}^{-1}$ versus $4.7 \times 10^{-2} \mathrm{~s}^{-1}$ at $353 \mathrm{~K}$ ). While both catalysts have the same initial TOF for 
butyraldehyde production, the $\operatorname{Pt}(1.7 \mathrm{~nm}) / \mathrm{SBA}-15$ is 1.5 times more active for the formation of crotyl alcohol, seven times more active for complete hydrogenation to butanol, and three times more active for decarbonylation at the onset of the reaction (Figure 4). The three-fold higher TOF for decarbonylation explains the larger extent of deactivation over $\mathrm{Pt}(1.7 \mathrm{~nm}) / \mathrm{SBA}-15$ compared to $\mathrm{Pt}(7.1 \mathrm{~nm}) / \mathrm{SBA}-15$. The initial TOF for decarbonylation (based on the appearance of propene and propane) over the four catalysts is $2.5 \times 10^{-3}, 1.8 \times 10^{-3}, 1.0 \times 10^{-3}$, and $1.0 \times 10^{-3} \mathrm{~s}^{-1}$ for $\mathrm{Pt}(1.7 \mathrm{~nm}) / \mathrm{SBA}-15$ (3.3-fold decrease in activity from initial-to-steady state), $\mathrm{Pt}(2.9 \mathrm{~nm}) / \mathrm{SBA}-15$ (2.3-fold decrease), Pt(3.6 nm)/SBA-15 (1.8-fold decrease), and Pt(7.1 nm)/SBA-15 (1.8-fold decrease), respectively (inset in Figure 4A). After the initial period of deactivation, the rate of decarbonylation is the same over the $\operatorname{Pt}(X) / \mathrm{SBA}-15$ catalysts. At longer time-onstream, the decreased activity of $\operatorname{Pt}(1.7 \mathrm{~nm}) / \mathrm{SBA}-15$ results from the formation of coke formation. The increased amount of decarbonylation and coke formation over the smaller $1.7 \mathrm{~nm}$ particles likely results from the increased C-C bond scission activity of lowcoordination sites that predominate on smaller nanoparticles.

\subsection{CO Poisoning}

$\mathrm{CO}$ poisoning was tested over the series of $\mathrm{Pt}(X) / \mathrm{SBA}-15$ catalysts by the addition of small quantities of $\mathrm{CO}$ to the feed. Reaction rates were measured over $\operatorname{Pt}(1.7 \mathrm{~nm}) / \mathrm{SBA}$ 15 and $\operatorname{Pt}(7.1 \mathrm{~nm}) / \mathrm{SBA}-15$ at $353 \mathrm{~K}$ as described above with the addition of a low pressure of $\mathrm{CO}(134-262 \mathrm{mTorr} \mathrm{CO})$. For both catalysts, the overall reaction rate decreased by $\sim 70 \%$ in the presence of 134 mTorr $\mathrm{CO}$ relative to the rate before the addition of $\mathrm{CO}$. The rate of formation of $\mathrm{C}_{3}$ hydrocarbons and butanol in the presence of $\mathrm{CO}$ was detectable, but too low for accurate determination of the TOF. The reaction 
order at $353 \mathrm{~K}$ in $\mathrm{CO}$ on the $\mathrm{Pt}(1.7 \mathrm{~nm}) / \mathrm{SBA}-15$ was -0.9 and -1.4 for butyraldehyde and crotyl alcohol, respectively, For the $\operatorname{Pt}(7.1 \mathrm{~nm}) / \mathrm{SBA}-15$, the reaction order in $\mathrm{CO}$ was 0.9 for both products (Figure 5). The negative reaction order in $\mathrm{CO}$ suggests it is the most abundant surface intermediate during the hydrogenation of crotonaldehyde in the presence of 130 - 260 mTorr $\mathrm{CO}$ [28]. The partial pressure of $\mathrm{CO}$ under reaction conditions (in the absence of $\mathrm{CO}$ ) due to decarbonylation ( $\sim 15$ mTorr based on the rate of $\mathrm{C}_{3}$ production) is much lower than the pressure of $\mathrm{CO}$ added through the $1 \% \mathrm{CO} / \mathrm{He}$ mixture. The reaction order for crotyl alcohol formation over $\operatorname{Pt}(1.7 \mathrm{~nm}) / \mathrm{SBA}-15$ is more negative than over $\operatorname{Pt}(7.1 \mathrm{~nm}) / \mathrm{SBA}-15$. While this is not completely understood, it appears to be consistent with the larger decrease in crotyl alcohol formation over $\operatorname{Pt}(1.7$ $\mathrm{nm}) / \mathrm{SBA}-15$

\subsection{Effect of Reduction Temperature on Catalyst Activity and Selectivity}

The effect of the reduction temperature was studied on $\operatorname{Pt}(X) / \mathrm{SBA}-15$ catalysts. All samples were calcined in $\mathrm{O}_{2}$ at high temperature in a horizontal tube furnace, followed by reduction in $\mathrm{H}_{2}$ (as described previously) in the U-tube reactor immediately prior to the hydrogenation of crotonaldehyde. All samples show an optimal reduction temperature of between 673 and $723 \mathrm{~K}$ with lower and higher reduction temperatures resulting in a decrease in the overall conversion rate of crotonaldehyde as well as a lower selectivity towards crotyl alcohol (Figure 6 shows this trend for $\operatorname{Pt}(7.1 \mathrm{~nm}) / \mathrm{SBA}-15)$. Particle sintering, which has been observed in many studies of Pt nanoparticles supported on $\mathrm{SiO}_{2}$ [29], is ruled out as the cause of these changes based on TEM observations of the catalysts before and after high temperature reduction. 
Residual carbon from the decomposition of PVP likely remains on the surface of the Pt nanoparticles after calcination and reduction. The amount and chemical state of this carbon will depend on the treatment time and temperature. Borodko et al. have shown that the carbonyl group of PVP interacts mores strongly with Pt NPs when oxidized in air at $363 \mathrm{~K}$ than when reduced in $\mathrm{H}_{2}$ at $363 \mathrm{~K}$ [30]. Coloma et al. studied the effect of reduction temperature for Pt/activated carbon catalysts in the hydrogenation of crotonaldehyde. They found that the type of oxygenated species on the support and the reduction temperature significantly changed the activity and selectivity of the catalyst [31]. The carbonyl groups in PVP may be similarly important for PVP stabilized Pt NP catalysts. The role of stabilizing molecules in catalysis is important and its effect on the catalytic performance of colloidally prepared NPs has not been adequately studied. Even after thermal treatment in $\mathrm{O}_{2}$ at $623-723 \mathrm{~K}$, some organic overlayer remains on the $\mathrm{Pt}$ surface and is modified by subsequent $\mathrm{H}_{2}$ treatment [30].

\section{Conclusions}

Monodisperse Pt nanoparticles $(1.7-7.1 \mathrm{~nm})$ synthesized by the reduction of chloroplatinic acid in alcohol containing poly(vinylpyrrolidone) were supported on mesoporous SBA-15 silica by nanoparticle encapsulation for the hydrogenation of crotonaldehyde. Solution phase synthesis of Pt nanoparticles provide excellent control over particle size and size distribution, allowing for better understanding of the effect of particle size on selectivity in catalysis. Both the activity and selectivity towards crotyl alcohol increased with increasing particle size. As the particle size increased from 1.7 to $7.1 \mathrm{~nm}$, the turnover frequency increased from $2.1 \times 10^{-2}$ to $4.8 \times 10^{-2} \mathrm{~s}^{-1}$ and the selectivity to crotyl alcohol increases from $14 \%$ to $34 \%$ (353 K and $\mathrm{H}_{2} /$ crotonaldehyde ratio of 20 ). 
The initial rate of decarbonylation is also particle size dependent: the TOF is $2.5 \times 10^{-3}$ over $\operatorname{Pt}(1.7 \mathrm{~nm}) / \mathrm{SBA}-15$ and $1.0 \times 10^{-3}$ over $\operatorname{Pt}(7.1 \mathrm{~nm}) / \mathrm{SBA}-15$. The increased rate of decarbonylation over catalysts with smaller Pt particles appears responsible for the increased deactivation. The reduction temperature significantly influenced both the activity and selectivity; there is an optimal reduction temperature of $673 \mathrm{~K}$ for $7.1 \mathrm{~nm} \mathrm{Pt}$ nanoparticle catalysts.

\section{Acknowledgements}

This work was supported by the Director, Office of Science, Office of Basic Energy Sciences, Division of Chemical Sciences, Geological and Biosciences of the U.S. Department of Energy under Contract No. DE-AC03-76SF00098. This work was also supported by the Director, Office of Science, Office of Basic Energy Sciences, Division of Materials Sciences and Engineering of the U.S. Department of Energy under Contract No. DE-AC02-05CH11231. R.M.R. acknowledges the Ford Motor Company for financial support through a graduate fellowship administered by the Berkeley Catalysis Center.

\section{References}

[1] J. D. Aiken and R. G. Finke, J. Mol. Catal. A: Chem. 145 (1999) 1.

[2] A. Roucoux, J. Schulz and H. Patin, Chem. Rev. 102 (2002) 3757.

[3] H. F. Lang, R. A. May, B. L. Iversen and B. D. Chandler, J. Am. Chem. Soc. 125 (2003) 14832.

[4] Z. Konya, V. F. Puntes, I. Kiricsi, J. Zhu, J. W. Ager, M. K. Ko, H. Frei, P. Alivisatos and G. A. Somorjai, Chem. Mater. 15 (2003) 1242. 
[5] H. Song, R. M. Rioux, J. D. Hoefelmeyer, R. Komor, K. Niesz, M. Grass, P. D. Yang and G. A. Somorjai, J. Am. Chem. Soc. 128 (2006) 3027.

[6] R. M. Rioux, H. Song, J. D. Hoefelmeyer, P. Yang and G. A. Somorjai, J. Phys. Chem. B 109 (2005) 2192.

[7] R. M. Rioux, J. D. Hoefelmeyer, M. Grass, H. Song, K. Niesz, P. D. Yang and G. A. Somorjai, Langmuir 24 (2008) 198.

[8] R. M. Rioux, R. Komor, H. Song, J. D. Hoefelmeyer, M. Grass, K. Niesz, P. D. Yang and G. A. Somorjai, J. Catal. 254 (2008) 1.

[9] M. E. Grass, Y. Yue, S. E. Habas, R. M. Rioux, C. I. Teall, P. Yang and G. A. Somorjai, J. Phys. Chem. C 112 (2008) 4797.

[10] P. Gallezot and D. Richard, Cat. Rev. - Sci. Eng. 40 (1998) 81.

[11] G. F. Santori, M. L. Casella, G. J. Siri, H. R. Aduriz and O. A. Ferretti, React. Kinet. Catal. Lett. 75 (2002) 225.

[12] A. Giroir-Fendler, D. Richard and P. Gallezot, Catal. Lett. 5 (1990) 175.

[13] M. Englisch, A. Jentys and J. A. Lercher, J. Catal. 166 (1997) 25.

[14] F. Delbecq and P. Sautet, J. Catal. 152 (1995) 217.

[15] A. B. Merlo, G. F. Santori, J. Sambeth, G. J. Siri, M. L. Casella and O. A. Ferretti, Catal. Commun. 7 (2006) 204.

[16] A. M. Venezia, L. F. Liotta, G. Pantaleo, V. La Parola, G. Deganello, A. Beck, Z. Koppany, K. Frey, D. Horvath and L. Guczi, Appl. Catal. A: Gen. 251 (2003) 359.

[17] D. S. Deutsch, A. Siani, P. T. Fanson, H. Hirata, S. Matsumoto, C. T. Williams and M. D. Amiridis, J. Phys. Chem. C 111 (2007) 4246.

[18] H. Song, F. Kim, S. Connor, G. A. Somorjai and P. D. Yang, J. Phys. Chem. B 109 (2005) 188.

[19] Y. Wang, J. Ren, K. Deng, L. Gui and Y. Tang, Chem. Mater. 12 (2000) 1622.

[20] T. Teranishi, M. Hosoe, T. Tanaka and M. Miyake, J. Phys. Chem. B 103 (1999) 3818.

[21] R. B. Grubbs, Polym. Rev. 47 (2007) 197 
[22] Y. K. Du, P. Yang, Z. G. Mou, N. P. Hua and L. Jiang, J. Appl. Poly. Sci. 99 (2006) 23.

[23] T. Birchem, C. M. Pradier, Y. Berthier and G. Cordier, J. Catal. 146 (1994) 503.

[24] A. Waghray and D. G. Blackmond, J. Phys. Chem. 97 (1993) 6002.

[25] U. K. Singh and M. A. Vannice, J. Catal. 191 (2000) 165.

[26] R. Shekhar and M. A. Barteau, Surf. Sci. 319 (1994) 298.

[27] P. Beccat, J. C. Bertolini, Y. Gauthier, J. Massardier and P. Ruiz, J. Catal. 126 (1990) 451.

[28] M. Boudart, AIChE J. 18 (1972) 465.

[29] R. T. K. Baker, E. B. Prestridge and R. L. Garten, J. Catal. 56 (1979) 390.

[30] Y. Borodko, S. M. Humphrey, T. D. Tilley, H. Frei and G. A. Somorjai, J. Phys. Chem. C 111 (2007) 6288.

[31] F. Coloma, A. Sepulveda-Escribano, J. L. G. Fierro and F. Rodriguez-Reinoso, Appl. Catal. A: Gen. 150 (1997) 165. 
Table 1: Physiochemical properties and summary of catalytic results for $\mathbf{P t}(\mathrm{X}) / \mathrm{SBA}-15$ catalysts

\begin{tabular}{|c|c|c|c|c|c|c|}
\hline Catalyst & $\begin{array}{c}\text { Pt Loading }{ }^{\mathrm{a}} \\
(\% \mathrm{Pt})\end{array}$ & $\begin{array}{c}\text { TEM } \\
\text { Size }(\mathbf{n m})\end{array}$ & $\mathbf{H}_{\text {tot }} / \mathbf{P t}^{\mathbf{b}}$ & $\begin{array}{c}\text { TOF } \\
\left(100 \times \mathrm{s}^{-1}\right)^{\mathrm{c}}\end{array}$ & $S_{\text {crotyl alcohol }}{ }^{d}$ & $\begin{array}{c}\mathbf{E}_{\mathrm{a}} \\
\left(\mathrm{kcal} \mathrm{mol}^{-1}\right)^{\mathrm{e}}\end{array}$ \\
\hline $\operatorname{Pt}(1.7 \mathrm{~nm}) / \mathrm{SBA}-15$ & 0.58 & 1.7 & 0.33 & 2.4 & 13.7 & 10.8 \\
\hline $\mathrm{Pt}(2.9 \mathrm{~nm}) / \mathrm{SBA}-15$ & 1.28 & 2.9 & 0.18 & 2.6 & 17.0 & 11.2 \\
\hline $\operatorname{Pt}(3.6 \mathrm{~nm}) / \mathrm{SBA}-15$ & 1.61 & 3.6 & 0.15 & 3.6 & 21.6 & 10.8 \\
\hline $\operatorname{Pt}(7.1 \mathrm{~nm}) / \mathrm{SBA}-15$ & 2.43 & 7.1 & 0.08 & 4.8 & 33.9 & 11.7 \\
\hline \multicolumn{7}{|c|}{$\begin{array}{l}\text { Loading determined by ICP-OES. } \\
\text { Total } \mathrm{H}_{2} \text { chemisorption at } 303 \mathrm{~K} \text {, extrapolated to } \mathrm{P}=0 \text {. } \\
\text { Overall steady-state crotonaldehyde hydrogenation rate per surface site at } 8 \text { Torr } \mathrm{C}_{6} \mathrm{H}_{4} \mathrm{O}, 160 \text { Torr } \mathrm{H}_{2} \text { and } \\
353 \mathrm{~K} \text {. } \\
\end{array}$} \\
\hline
\end{tabular}




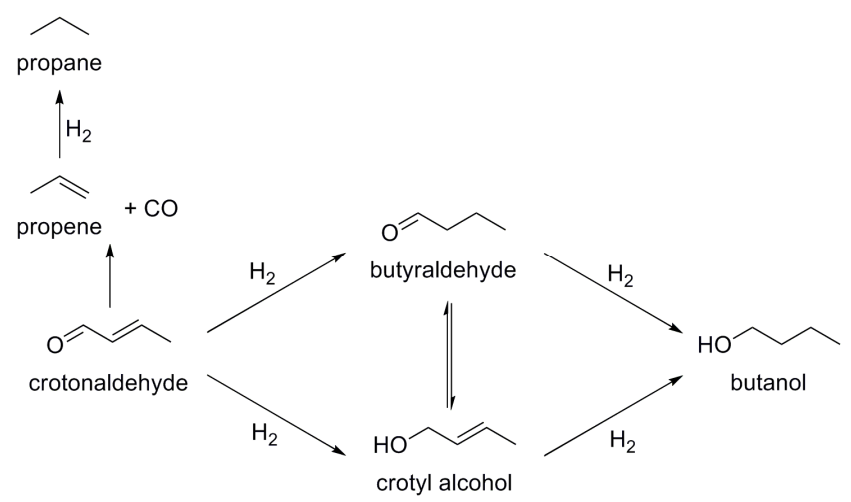

Scheme 1. Reaction network for the conversion of crotonaldehyde in hydrogen. 


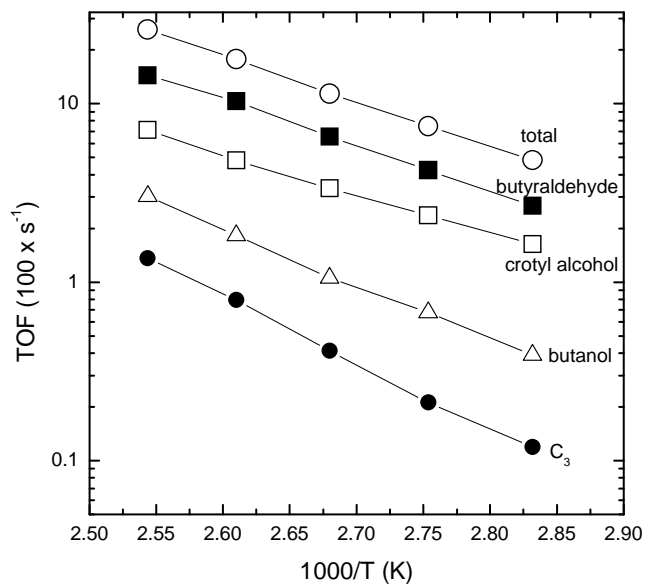

Figure 1. Turnover frequency for the disappearance of crotonaldehyde $(0)$ and formation of butyraldehyde $(\boldsymbol{\square})$, crotyl alcohol $(\square)$, butanol $(\Delta)$, and $\mathrm{C}_{3}$ hydrocarbons $(\bullet)$ as an Arrhenius plot for $\operatorname{Pt}(7.1 \mathrm{~nm}) / \mathrm{SBA}-15$. The pressure of crotonaldehyde and hydrogen was 8 Torr and 160 Torr (760 Torr total pressure). The apparent activation energy is 12 $\mathrm{kcal} \mathrm{mol}^{-1}$ (overall), $17 \mathrm{kcal} \mathrm{mol}^{-1}$ (butyraldehyde), $10 \mathrm{kcal} \mathrm{mol}^{-1}$ (crotyl alcohol), $14 \mathrm{kcal}$ $\mathrm{mol}^{-1}$ (butanol), and $12 \mathrm{kcal} \mathrm{mol}^{-1}\left(\mathrm{C}_{3}\right.$ hydrocarbons). 


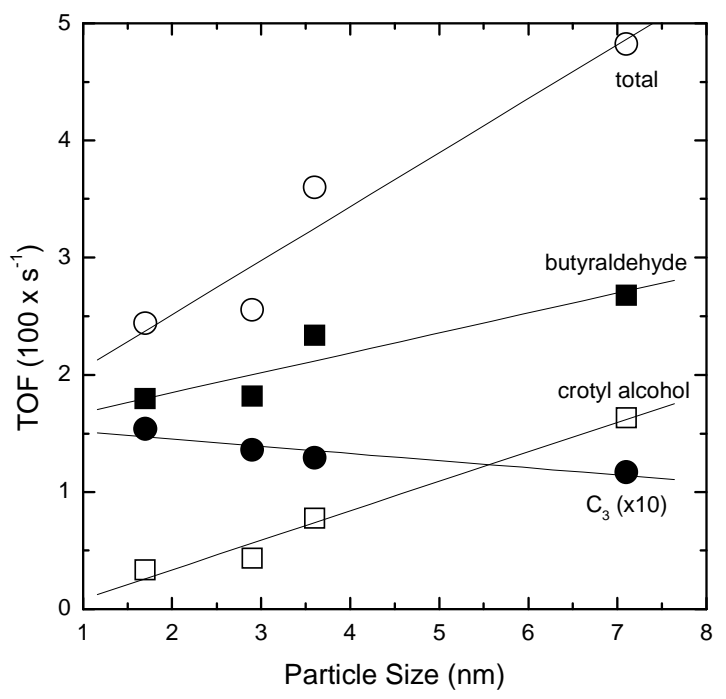

Figure 2: Selectivity as a function of particle size at $353 \mathrm{~K}$ for butyraldehyde ( $\mathbf{\square})$, crotyl

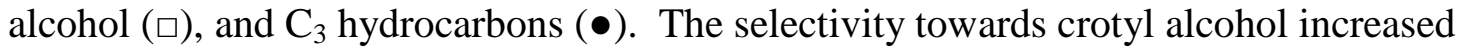
with increasing particle size, while the selectivity to butyraldehyde decreased. The pressure of crotonaldehyde and hydrogen was 8 Torr and 160 Torr (760 Torr total pressure). 


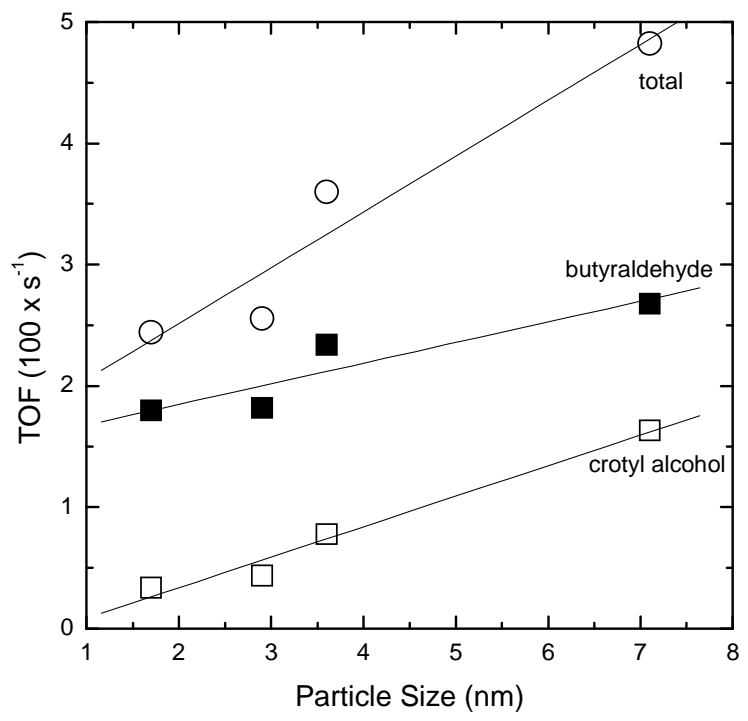

Figure 3: Turnover frequency as a function of particle size at $353 \mathrm{~K}$ for butyraldehyde (匹) and crotyl alcohol (口). The overall TOF (०) increases with particle size, but more strongly for the formation of crotyl alcohol. The pressure of crotonaldehyde and hydrogen was 8 Torr and 160 Torr (760 Torr total pressure). 

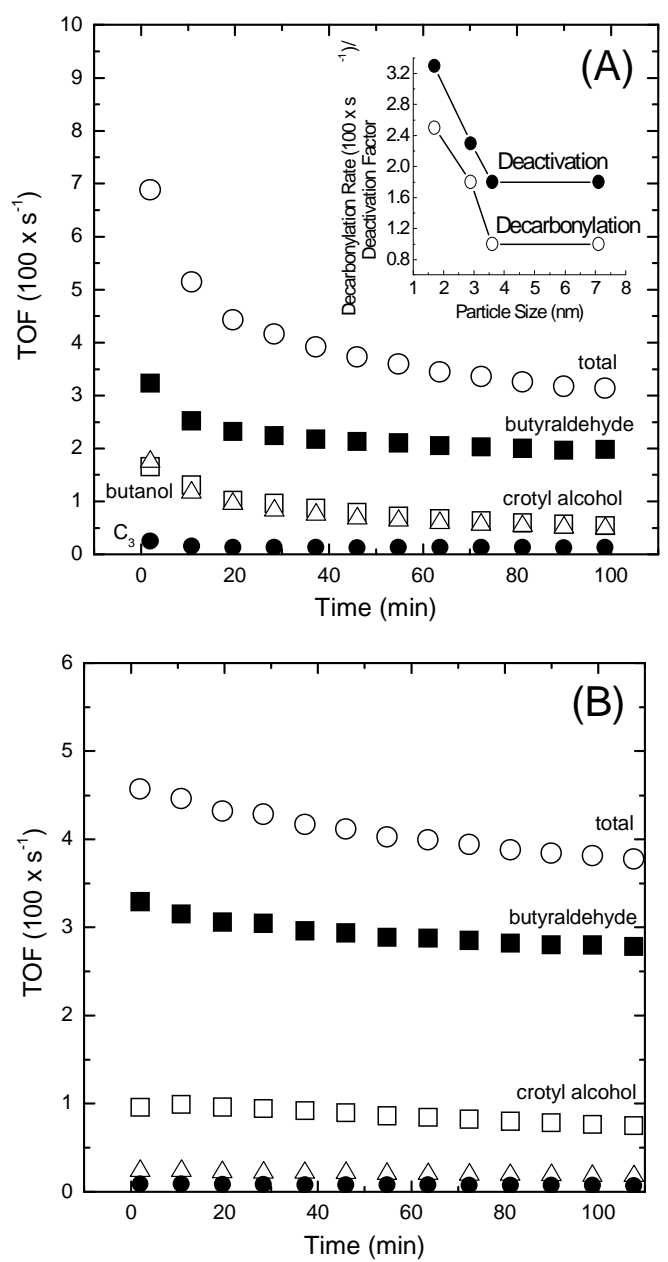

Figure 4. Initial deactivation of (A) $\mathrm{Pt}(1.7 \mathrm{~nm}) / \mathrm{SBA}-15$ and $(\mathrm{B}) \operatorname{Pt}(7.1 \mathrm{~nm}) / \mathrm{SBA}-15$ with time-on-stream at $353 \mathrm{~K}$. The initial overall turnover frequency is greater for the $1.7 \mathrm{~nm}$ particles, but they demonstrate a greater extent of deactivation (presumably due to the greater rate of decarbonylation). The disappearance of crotonaldehyde (०) and the formation of butyraldehyde ( $\square)$, crotyl alcohol $(\square)$, butanol $(\Delta)$, and $\mathrm{C}_{3}$ hydrocarbons $(\bullet)$ are shown. The inset shows the initial rate of decarbonylation $(\circ)$ and the amount of deactivation $(\bullet)$, where the deactivation factor is expressed as initial rate/steady state rate for the disappearance of crotonaldehyde. The pressure of crotonaldehyde and hydrogen was 8 Torr and 160 Torr (760 Torr total pressure). 

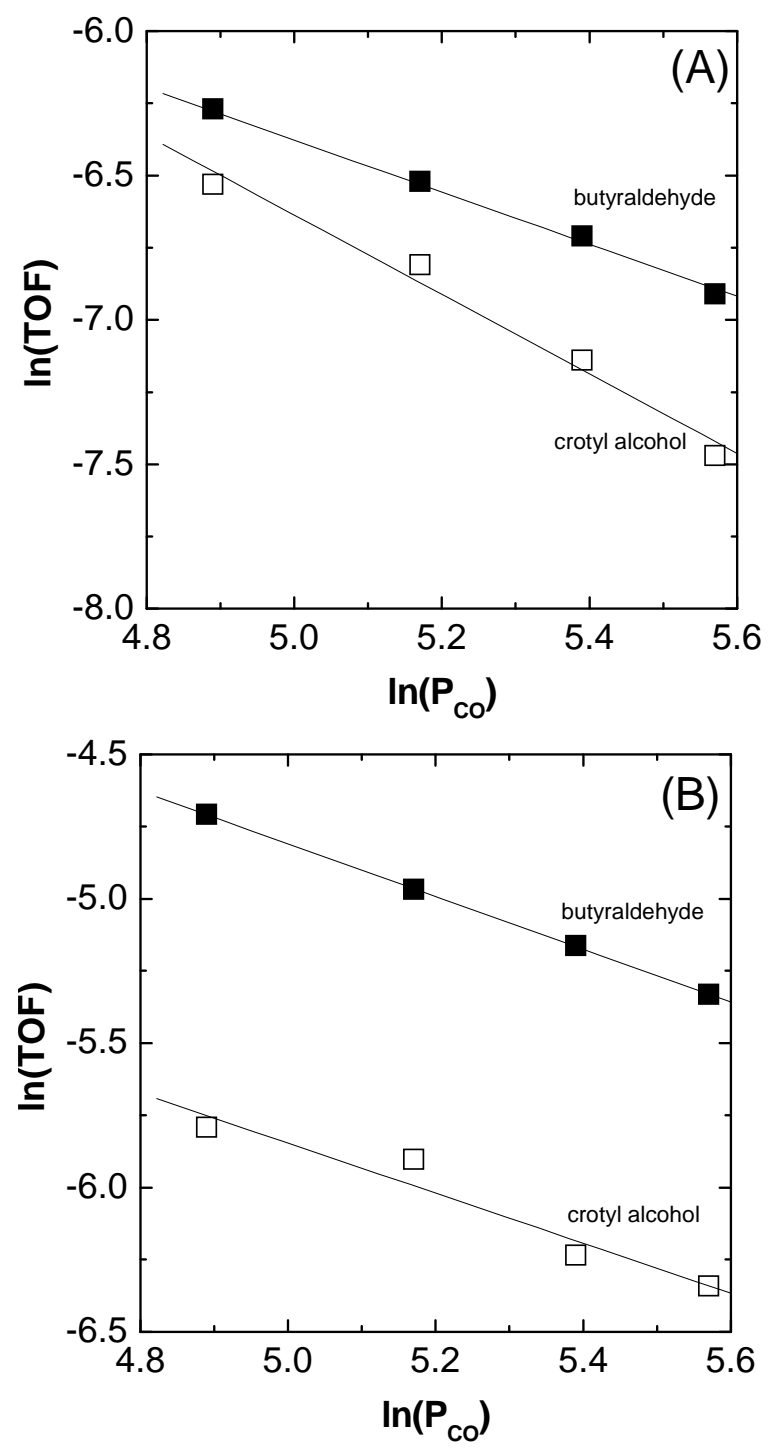

Figure 5. The dependence of the rate of butyraldehyde ( $\square$ ) and crotyl alcohol ( $\square$ ) formation on the pressure of $\mathrm{CO}$ for $(\mathrm{A}) \operatorname{Pt}(1.7 \mathrm{~nm}) / \mathrm{SBA}-15$ and $(\mathrm{B}) \operatorname{Pt}(7.1 \mathrm{~nm}) / \mathrm{SBA}-15$. The reaction conditions were $353 \mathrm{~K}, 8$ Torr crotonaldehyde, 160 Torr $\mathrm{H}_{2}, 134-262$ mTorr $\mathrm{CO}$ in a balance He. 


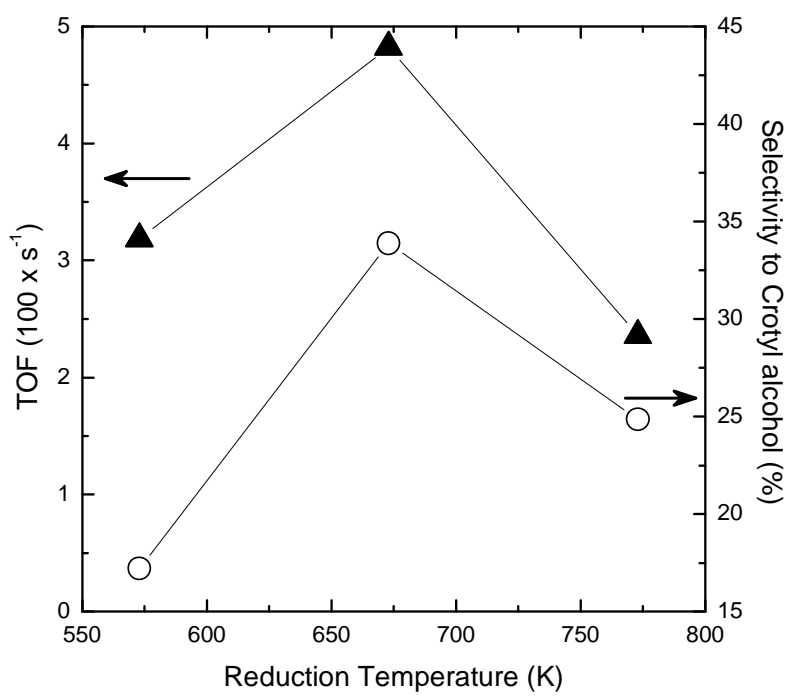

Figure 6. Overall turnover frequency ( $\boldsymbol{\Delta}$ ) and selectivity (o) to crotyl alcohol at $353 \mathrm{~K}$ over $\operatorname{Pt}(7.1 \mathrm{~nm}) / \mathrm{SBA}-15$ as a function of the reduction temperature (after calcination at $723 \mathrm{~K}$ for $24 \mathrm{~h}$ ). The overall TOF is maximized after reduction at $673 \mathrm{~K}$. The pressure of crotonaldehyde and hydrogen was 8 Torr and 160 Torr (760 Torr total pressure). 